\title{
Jasmonic Acid and Ethylene Participate in the Gibberellin-Induced Ovule Programmed Cell Death Process in Seedless Pear '1913' (Pyrus hybrid)
}

\author{
Huibin Wang ${ }^{1,2}$, Shichao Zhang ${ }^{1,2}$, Yingying $Q u^{1,2}$, Rui Gao ${ }^{1,2}$, Yuxiong Xiao ${ }^{1,2}$, Zhigang Wang ${ }^{1,2, *}$, \\ Rui Zhai ${ }^{1,2}$, Chengquan Yang ${ }^{1,2}$ and Lingfei $\mathrm{Xu}{ }^{1,2, *}$ \\ 1 College of Horticulture, Northwest A\&F University, Taicheng Road. 3, Yangling, Xianyang 712100, China; \\ wanghuibin069@nwsuaf.edu.cn (H.W.); woshizsc@nwsuaf.edu.cn (S.Z.); 2019050262@nwsuaf.edu.cn (Y.Q.); \\ hopppie@nwsuaf.edu.cn (R.G.); yuxiongx@nwsuaf.edu.cn (Y.X.); Zhai.Rui@nwsuaf.edu.cn (R.Z.); \\ cqyang@nwsuaf.edu.cn (C.Y.) \\ 2 State Key Laboratory of Crop Stress Biology for Arid Areas, Northwest A\&F University, Taicheng Road, \\ Yangling, Xianyang 712100, China \\ * Correspondence: wzhg001@nwsuaf.edu.cn (Z.W.); lingfeixu@nwsuaf.edu.cn (L.X.); \\ Tel.: +86-29-8708-1023 (L.X.); Fax: +86-29-8708-2613 (L.X.)
}

\section{check for}

updates

Citation: Wang, H.; Zhang, S.; Qu, Y.; Gao, R.; Xiao, Y.; Wang, Z.; Zhai, R.; Yang, C.; Xu, L. Jasmonic Acid and Ethylene Participate in the Gibberellin-Induced Ovule Programmed Cell Death Process in Seedless Pear '1913' (Pyrus hybrid). Int. J. Mol. Sci. 2021, 22, 9844. https://doi.org/10.3390/ijms22189844

Academic Editor:

Jutta Ludwig-Müller

Received: 15 July 2021

Accepted: 8 September 2021

Published: 11 September 2021

Publisher's Note: MDPI stays neutral with regard to jurisdictional claims in published maps and institutional affiliations.

Copyright: (c) 2021 by the authors. Licensee MDPI, Basel, Switzerland. This article is an open access article distributed under the terms and conditions of the Creative Commons Attribution (CC BY) license (https:// creativecommons.org/licenses/by/ $4.0 /)$

\begin{abstract}
Seedless fruit is a feature appreciated by consumers. The ovule abortion process is highly orchestrated and controlled by numerous environmental and endogenous signals. However, the mechanisms underlying ovule abortion in pear remain obscure. Here, we found that gibberellins (GAs) have diverse functions during ovules development between seedless pear '1913' and seeded pear, and that $\mathrm{GA}_{4+7}$ activates a potential programmed cell death process in '1913' ovules. After hormone analyses, strong correlations were determined among jasmonic acid (JA), ethylene and salicylic acid (SA) in seedless and seeded cultivars, and $\mathrm{GA}_{4+7}$ treatments altered the hormone accumulation levels in ovules, resulting in significant correlations between GA and both JA and ethylene. Additionally, SA contributed to ovule abortion in '1913'. Exogenously supplying JA, SA or the ethylene precursor 1-aminocyclopropane-1-carboxylic acid promoted 'Bartlett' seed death. The regulatory mechanism in which ethylene controls ovule death has been demonstrated; therefore, JA's role in regulating '1913' ovule abortion was investigated. A further study identified that the JA signaling receptor MYC2 bound the SENESCENCE-ASSOCIATED 39 promoter and triggered its expression to regulate ovule abortion. Thus, we established ovule abortion-related relationships between GA and the hormones JA, ethylene and SA, and we determined their synergistic functions in regulating ovule death.
\end{abstract}

Keywords: seedless pear; GA; JA; SA; ethylene; ovule; PCD; PbSAG39

\section{Introduction}

Pear as an important fruit, both nutritionally and commercially, and is planted worldwide. Presently, seedless fruit is desired by consumers, and various seedless species, including grapes and pears, have been bred in the past several years [1-3]. The fresh fruit of seedless pear has a high nutritional quality and is more convenient to consume. Thus, identifying the genetic mechanisms and key regulatory genes involved in seedless fruit production is important for pear breeding and meeting market demand. In seeded plants, abnormal fertilization, female and male sterility, embryo abortion, hormone regulation and various other factors cause seedlessness [4]. In a previous study, we identified the seedless pear variety '1913', in which seedlessness is caused by failed fertilization. After pollination, a potential programmed cell death (PCD) mechanism in ovules is triggered in '1913' [4]. Although seed abortion has been studied, there are still many unknown details.

As a basic cellular process, PCD has adopted a series of vital functions in multicellular organisms. In individual organisms, the gene coding procedures associated with aging and 
death control generational replacement, which is the driving force of adaptive evolution. In plants, there are various forms of PCD, and they are important in development and in abiotic and biotic stress responses. During reproductive and vegetative development, development-controlled PCD (dPCD) occurs and plays key roles. It often ends senescence or the functions of no longer required cells [5]. Additionally, different dPCD events are distinguishable on the basis of the developmental context. Senescence is best-defined as the process of growing old. In plant biology, senescence is often used to describe the reproducible patterns of cell death and biochemical changes, and it is considered to be a type of PCD [6].

In plants, various hormones participate in PCD processes. For instance, ethylene, jasmonic acid (JA) and salicylic acid (SA) have been implicated in PCD signaling [7-10]. Among them, ethylene is the best-characterized dPCD hormone. Ethylene has been described as an important regulator in controlling the early PCD process in plants, for example, ethylene signaling is required for synergid degeneration [11], nucellus PCD [12], endosperm development [13] and ovule senescence [14]. Additionally, ETHYLENE-INCENSITIVE 3-LIKE (EIL1), the receptor of ethylene signaling, directly activates the expression of the senescence-related gene CYSTEINE PROTEINASE 1 (Cysp1) to regulate ovule senescence in pear [4]. Another important hormone in plant development, JA, is essential for plant defense and development, and it serves as an important signal that triggers senescence-associated gene expression and tissue senescence [8]. MYELOCYTOMATOSIS (MYC) transcription factors (MYC2, 3 and 4) function as the general switches in the JA-signaling pathway, and they negatively regulate cell cycle-related gene expression and inhibit plant growth $[15,16]$. In Arabidopsis, MYC2 directly binds the promoter and activates the expression of SENECENCE-ASSOCIATED GENE 29 (SAG29) to trigger JA-induced leaf senescence [8]. However, there are limited reports on the relationship between JA and ovule senescence. In plants, ovules are derived from specialized meristematic regions within the carpels, referred to as the placentas, and carpels are proposed to have evolved from ancestral foliar organs, either leaf-like sporophylls that folded to enclose the ovules or bract-like structures that subtend shoot-like ovules [17]. Additionally, a gene homologous to the Arabidopsis leaf senescence-associated gene SAG12 in pear, SAG39 (LOC103945424), is expressed in ovules [4]. Thus, there may be similar regulatory mechanisms between leaf and ovule senescence. In plants, SA, which has been widely studied in disease resistance, including pathogen-mediated response and cell death, may be involved in senescenceassociated cell death [18]. Evidence indicates that SA is also associated with leaf senescence. When leaves are undergoing senescence, there is a high SA level, and the npr 1 and pad4 mutants, which are defective in SA signaling, show reduced expression levels of a number of $S A G$ genes, including $S A G 12$ in Arabidopsis [19]. Additionally, SA's regulation of gene expression during developmental senescence has been reported by Buchanan-Wollaston et al. [20].

Hormones function as important mediators in the regulation of seed development and abortion. In the enlightenment period of seed development, the biosynthesis of various hormones, such as auxin and gibberellin (GA), is activated, and hormones appear to have prominent roles in synchronizing fertilization and ovule growth [21]. In Arabidopsis, pollination triggers the biosynthesis of auxin in ovules, and then auxin signaling promotes active GA biosynthesis in ovules, which promotes ovule development [21]. As an important hormone, GA plays fundamental roles in various plant developmental processes, including ovule development. The ectopic expression of pea GA inactivates the enzyme 2-oxidase in Arabidopsis, causing seed abortion [22]. In pea, mutations of the GA biosynthesis genes LH1 and $\mathrm{LH} 2$ greatly affect embryogenesis and cause the production of abnormal seeds [23]. Thus, GAs appear to positively regulate ovule development at early stages. Therefore, determining whether GA applications could block seed abortion in seedless pear is of interest.

In this study, we found that GA played different roles in the ovule development of seeded and seedless pear. $\mathrm{A} \mathrm{GA}_{4+7}$ treatment triggered a potential PCD process in seedless 
pear, and we determined that JA and ethylene participate in GA-induced ovule abortion. In addition, SA is involved in pear ovule abortion, although it did not respond to the $\mathrm{GA}_{4+7}$ treatment. Furthermore, a link between JA signaling and pear ovule abortion was established, in which PbMYC2 directly mediates the expression of PbSAG39, leading to ovule abortion. These results provide a meaningful foundation for understanding ovule abortion and are useful for seedless fruit breeding.

\section{Results}

\section{1. $G A_{4+7}$ Advances the PCD Process of ' 1913 ' Unpollinated Ovules}

To identify the relationship between GA and seedless pear ovule abortion, we sprayed $\mathrm{GA}_{4+7}$ on unpollinated ovaries of '1913', 'Bartlett' and 'Dangshansu' at the full-bloom stage. The morphological observations revealed that $\mathrm{GA}_{4+7}$-treated '1913' ovules began to brown at 8 days after treatment (DAT), and the whole ovule turned completely brown or black by 16 DAT. On the contrary, the $\mathrm{GA}_{4+7}$-treated ovules of seeded pears 'Bartlett' and 'Dangshansu' developed normally until 16 DAT (Figure 1A). However, the solvent-treated ovules of the three pear varieties were normal at $8 \mathrm{DAT}$, and small areas on the ovules began to brown by 16 DAT (Figure 1A).

\section{A}

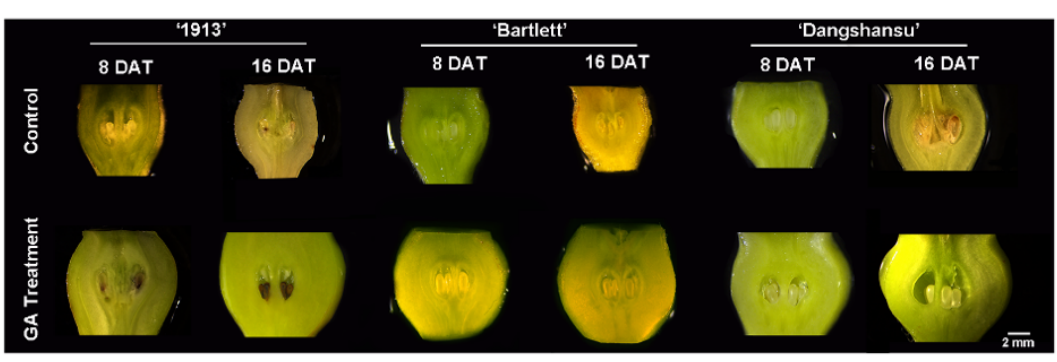

B

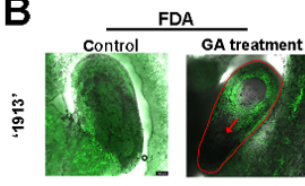

喜

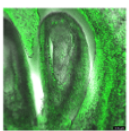

势

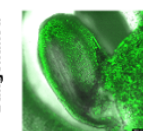

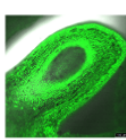

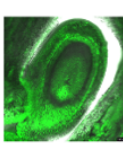

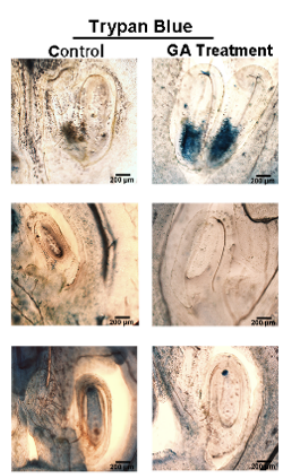

C ${ }_{50}^{50} \square$ Control $\square$ GA $_{4+7}$ treatment
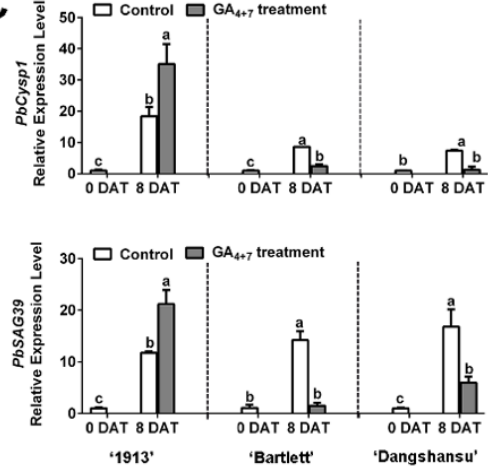

Figure 1. Ovule development of samples taken at 8 and 16 days after treatment (DAT) from '1913', 'Bartlett' and 'Dangshansu'. (A) Observations of ovule development at 8 and 16 DAT. (B) The ovules of 8 DAT from '1913', 'Bartlett' and 'Dangshansu' were stained by FDA and trypan blue. (C) Expression profile of ovule senescence-related genes PbCysp1 and PbSAG39 in ovules of 0 and 8 DAT from '1913', 'Bartlett' and 'Dangshansu'. The sample of 0 DAT means the sample was collected before treatment. Control: unpollinated ovules with solvent treatment. GA Treatment: $\mathrm{GA}_{4+7}$ treatment. The data are mean \pm SD of three biological replicates. Error bars represent the standard deviation. Different lowercase letters indicate significant difference at $p<0.05$ (Duncan's multiple range test).

Then, ovule cell development was observed at 8 DAT using FDA and trypan bluestaining assays. There were no fluorescence signals, and obvious blue staining was observed at the adaxial-funiculi of '1913' GA $4+7$-treated ovules, whereas the other treated ovules had obvious fluorescence signals but were not stained by trypan blue (Figure 1B). These observations indicate that the '1913' ovules were dead at 8 DAT with GA ${ }_{4+7}$. To further investigate, we analyzed the expression profiles of $P b C y s p 1$ and PbSAG39, which are genes in pear homologous to Cysp1 in Brassica napus and SAG12 in Arabidopsis, respectively. 
Cysp1 and SAG12 are marker genes of PCD and participate in ovule senescence [14,24]. The qRT-PCR indicated that $\mathrm{GA}_{4+7}$ increased the expression levels of $P b C y s p 1$ and $P b$ SAG39 in '1913' ovules at 8 DAT, but it reduced their expression levels in 'Bartlett' and 'Dangshansu' ovules, compared with the solvent-treated ovules (Figure 1C). In comparison to the expression levels of the two marker genes in solvent-treated ovules at $0 \mathrm{DAT}$, the levels significantly increased in the three pear varieties by $8 \mathrm{DAT}$, which suggested that the ovules underwent a PCD process when not pollinated at the full-bloom stage. Additionally, $\mathrm{GA}_{4+7}$ appeared to promote the PCD process in '1913' ovules and inhibit the PCD process in 'Bartlett' and 'Dangshansu', which is consistent with the morphological observations and staining assays. Thus, $\mathrm{GA}_{4+7}$ may trigger a potential PCD process in the seedless '1913' pear variety.

\subsection{SA, JA and Ethylene Enriched in '1913' Ovules}

To determine why the $\mathrm{GA}_{4+7}$ treatment had different effects on ovule development between seedless and seeded pears, endogenous hormones were quantified in the ovules of '1913', 'Bartlett' and 'Dangshansu' at 0 and 8 DAT. By analyzing the solvent-treated ovules in the three pear varieties at $8 \mathrm{DAT}$, the results showed that the levels of 1-aminocyclopropane1-carboxylic acid (ACC), which is the precursor of ethylene, JA and SA were significantly different in seeded and seedless ovules at $8 \mathrm{DAT}$, and their concentrations were greater in the ovules of '1913' than in 'Bartlett' and 'Dangshansu'. A similar difference was also observed in $\mathrm{GA}_{4+7}$-treated ovules, but in contrast, the contents of CTK, IAA and ABA did not have absolutely consistent differences between seeded and seedless ovules (Figure 2). Moreover, $\mathrm{GA}_{4+7}$ treatments increased the content of ACC, JA and IAA in three pear ovules at $8 \mathrm{DAT}$, and $\mathrm{ABA}$ content was reduced by $\mathrm{GA}_{4+7}$ treatments, compared with the solvent-treated ovules (Figure 2). Furthermore, by comparing the hormone content in solvent-treated ovules between 0 and $8 \mathrm{DAT}$, we found that the upregulated levels of ACC, JA and SA in '1913' ovules by 8 DAT were more significant than those in seeded pear ovules (Figure 2). These results suggest that ethylene, JA and SA may be involved in the death of '1913' ovules and the increased level of ethylene and JA by $\mathrm{GA}_{4+7}$ treatment may contribute to the $\mathrm{GA}_{4+7}$-induced '1913' ovule abortion.
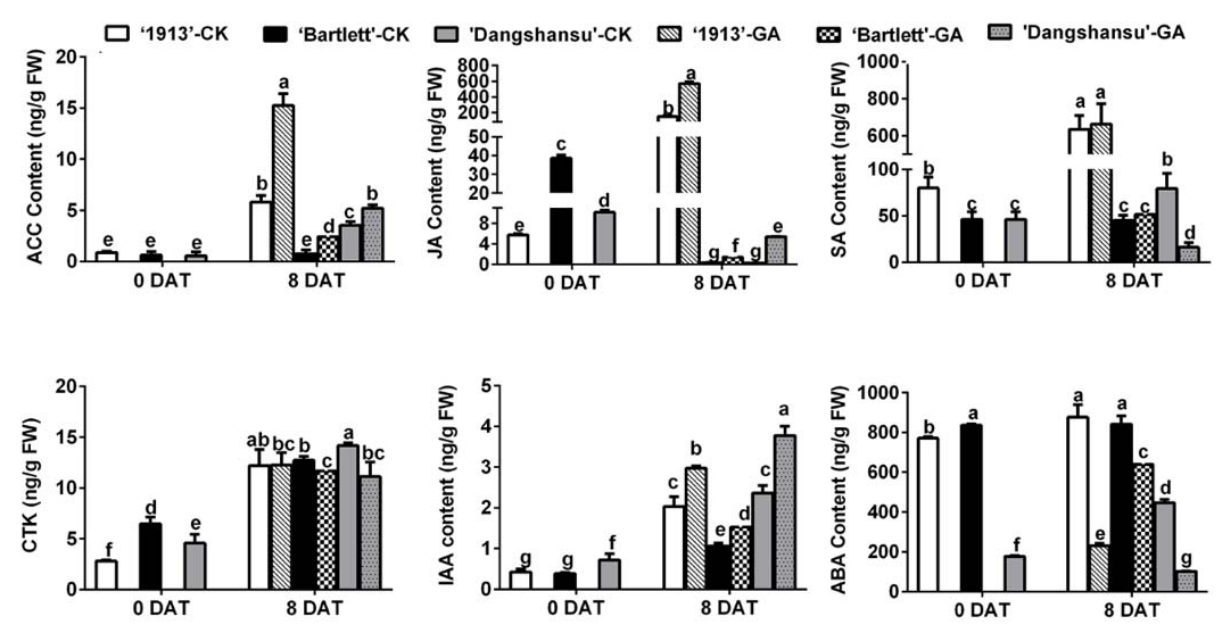

Figure 2. Hormone content of ovules at 0 and 8 DAT from '1913', 'Bartlett' and 'Dangshansu', including ACC (aminocyclopropane-1-carboxylic acid, the precursor of ethylene), JA, SA, CTK (cytikinins), IAA and ABA. The samples at 8 DAT were collected from ovules with $\mathrm{GA}_{4+7}$ treatment and untreated. DAT, days after treatment. The data are mean \pm SD of three biological replicates. Error bars represent the standard deviation. Different lowercase letters indicate significant difference at $p<0.05$ (Duncan's multiple range test). 


\section{3. $G A_{4+7}$ Treatment Increases the Expression Levels of JA and Ethylene Pathway-Related Genes}

Ethylene, JA and SA may participate in '1913' ovule death, and in order to determine the relationship between candidate hormones ethylene, SA, JA and $\mathrm{GA}_{4+7}$ treatment, the expression of their biosynthesis and signaling response-related genes were assessed by qRT-PCR. Comparing the solvent treatment (control) and $\mathrm{GA}_{4+7}$ treatment groups at 8 DAT, we found that $\mathrm{GA}_{4+7}$ treatment increased the expression levels of PbACO1, PbACS1, PbEIL1, PbAOC1, PbLOX3 and PbMYC2, and the differences in '1913' were more significant than those in 'Bartlett' and 'Dangshansu' (Figure 3). Consistent with the hormone level changes (Figure 2), there were no differences in the expression levels of SA synthesis genes (PbEDS1 and PbSARD) and a signaling response-related gene (PbNPR1) between $\mathrm{GA}_{4+7}$-treated and control groups (Figure 3 ). These data collectively indicate that the $\mathrm{GA}_{4+7}$ treatment promoted the biosynthesis and signaling transduction of ethylene and JA, which accelerated the ovule death process in '1913'.
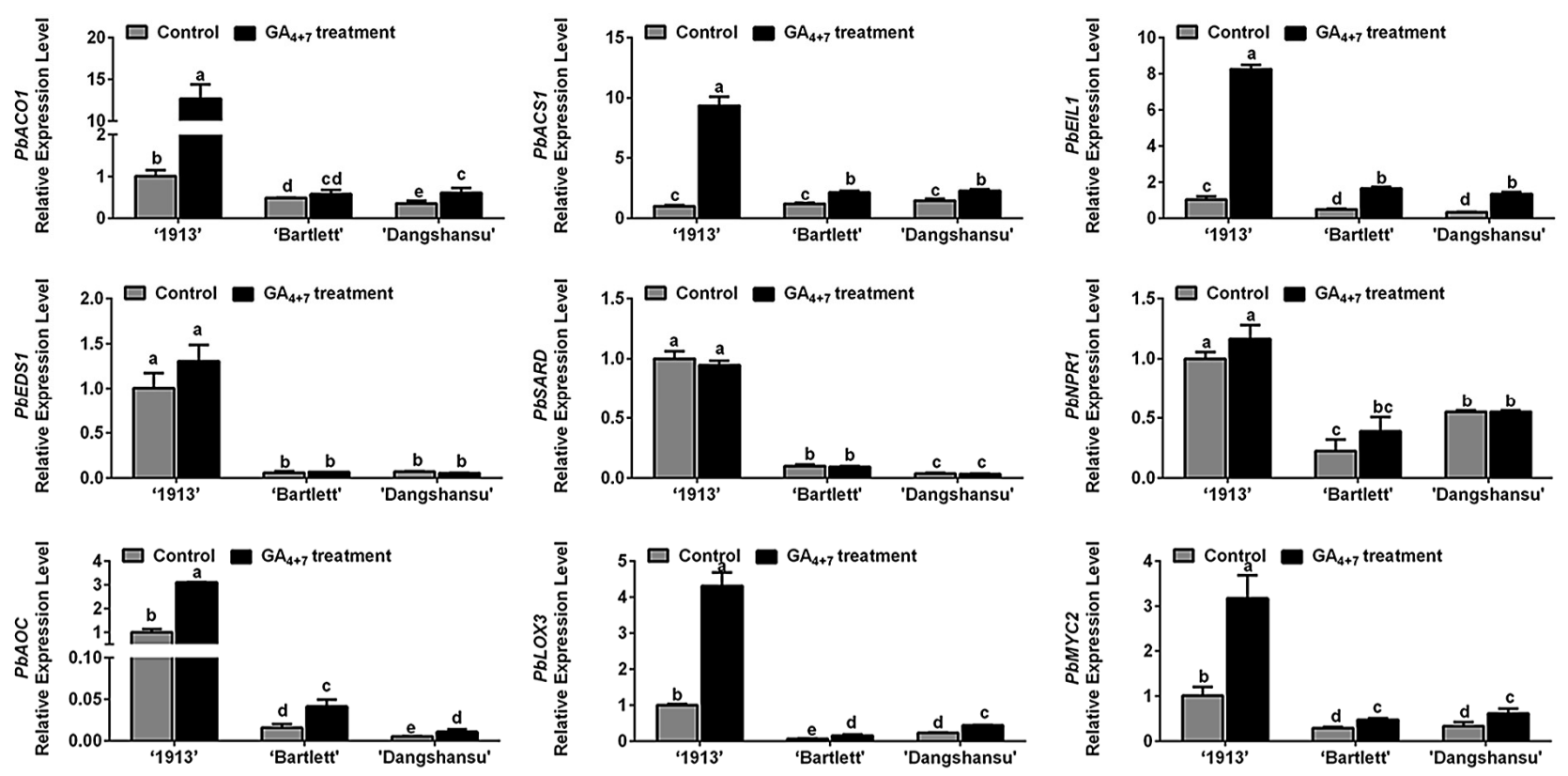

Figure 3. Relationship analyses between ethylene, JA and SA with $\mathrm{GA}_{4+7}$ treatment. The expression profile analyses of ethylene, JA, SA synthesis and signaling response-related genes between $\mathrm{GA}_{4+7}$-treated and untreated ovules at 8 DAT. The data are mean $\pm \mathrm{SD}$ of three biological replicates. Error bars represent the standard deviation. Different lowercase letters indicate significant difference at $p<0.05$ (Duncan's multiple range test).

\subsection{Differences in Ethylene and JA Pathway Gene Enrichments Result in Different Cell Death-Gradient Orientations between '1913' and Seeded Pears}

Observations of unpollinated ovule development at 16 DAT revealed a difference between seedless and seeded pear. We collected the unpollinated ovules with solvent treatment and found that ovule death in '1913' fist occurred at the bottom, whereas in 'Bartlett' and 'Dangshansu' pear, it occurred at the top (Figure 4A). The ovules were then divided into top and bottom samples. An analysis of the expression levels of ethylene, JA and SA pathway genes in '1913' revealed that the levels of PbACO1, PbEIL1, PbAOC and $P b M Y C 2$ were higher at the bottoms of ovules than at that the tops (Figure 4B). After analyzing the ethylene- and JA-related genes in 'Bartlett' and 'Dangshansu', we confirmed that their expression differences, between the tops and bottoms of ovules, were opposite to those in '1913' ovules (Figure 4B). The samples consisted of solvent-treated ovules at 16 DAT from '1913', 'Bartlett' and 'Dangshansu'. In addition, there were no significant differences in the expression gradients of SA-related genes, including PbEDS1 and PbNPR1 
(Figure 4B). The data further suggest that ethylene and JA are involved in the death of seedless pear ovules.

\section{A}

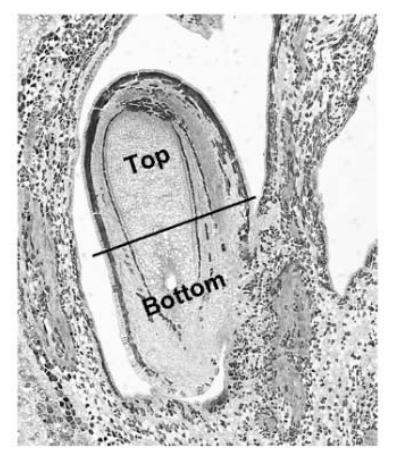

B
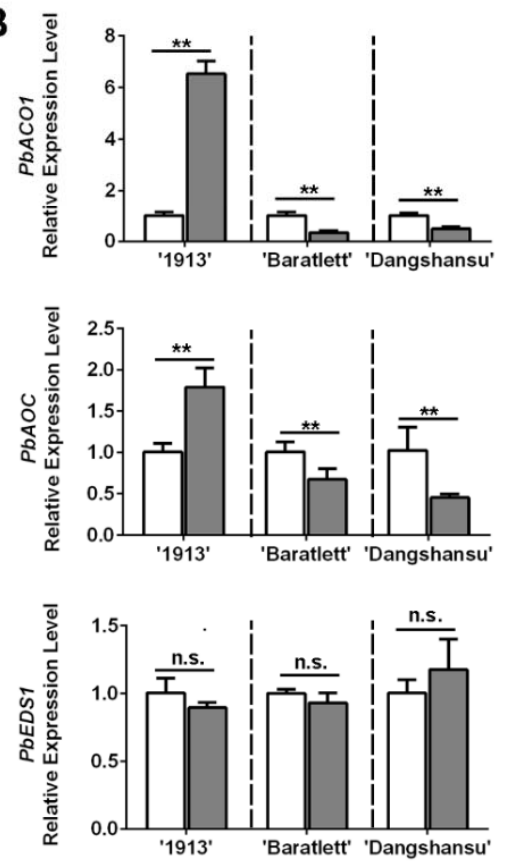

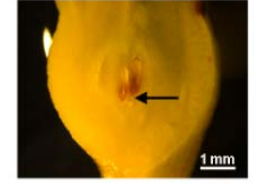

' 1913 '

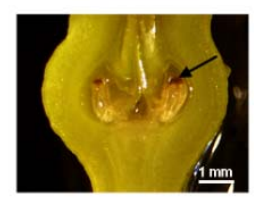

'Dangshansu'

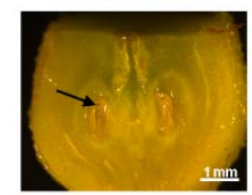

'Bartlett'
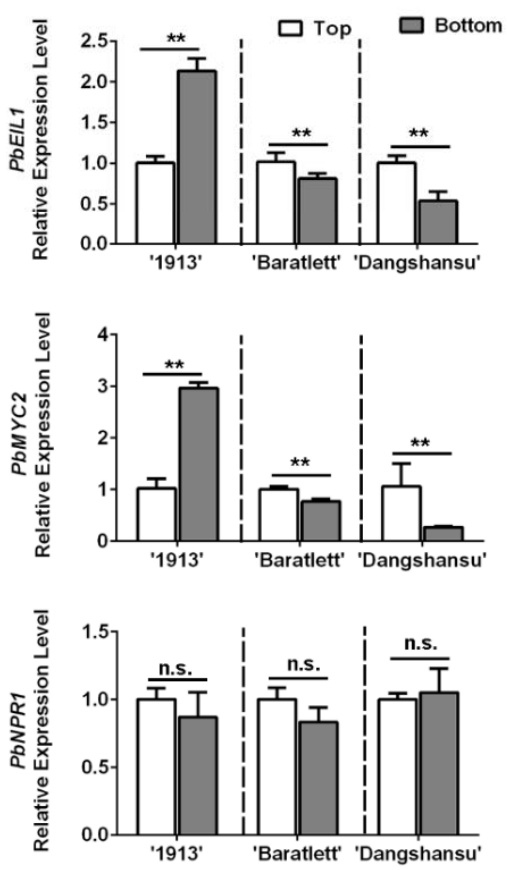

Figure 4. The distribution of JA, SA and ethylene in ovules of seedless pear '1913' and seeded pear 'Bartlett' and 'Dangshansu' is different. (A) The left pear ovule is divided into two parts, the top (the abaxial-funiculus tissue) and bottom (the adaxial-funiculus tissue). Right exhibits that the unpollinated ovules of '1913', 'Bartlett' and 'Dangshansu' at 16 DAT show different locations of ovule cell death. The black arrows point to areas of significant ovule cell death. (B) The expression level of ethylene, JA and SA pathway-related genes on the top and bottom ovule tissue from the seeded and seedless pear. The samples were collected from unpollinated ovules at 16 DAT. The expression level of each gene in the top sample was normalized to 1.0. The data are mean \pm SD of three biological replicates. Error bars represent the standard deviation. Asterisks indicate significant difference at ** $p<0.01$ (Student's $t$-test). n.s., no significance.

\subsection{Ethylene, JA and SA Treatments Increased the Expression of PCD-Related Genes}

To further determine the relationships between ethylene, JA and SA levels and ovule death in pear, we generated high-hormone level samples by spraying ACC, JA and SA independently on 'Bartlett' pollinated ovules in vitro. At 4 DAT, the ACC-, JA- and SAtreated ovules showed obvious browning on their surfaces, compared with the control (Figure 5A). Using trypan blue staining, we observed that obvious tissue death occurred in the hormone-treated ovules (Figure 5A), which indicated that each of the three hormone treatments increased ovule death. Then, we analyzed the PbNPR1, PbMYC2 and PbEIL1 expression levels independently in SA-, JA- and ACC-treated samples. Their increased ex- 
pression levels indicated that the three hormones function in ovules (Figure 5B). In addition, the individual SA, JA and ACC treatments increased the expression levels of senescenceassociated genes in the ovules (Figure $5 \mathrm{C}$ ). These results suggest that SA, JA and ethylene affect pear ovule PCD by increasing the expression levels of the cell death-related genes. Thus, we confirmed that ethylene and JA participate in the GA-induced advanced abortion of '1913' ovules and that SA may also be involved, although it did not respond to the $\mathrm{GA}_{4+7}$ treatment.

A

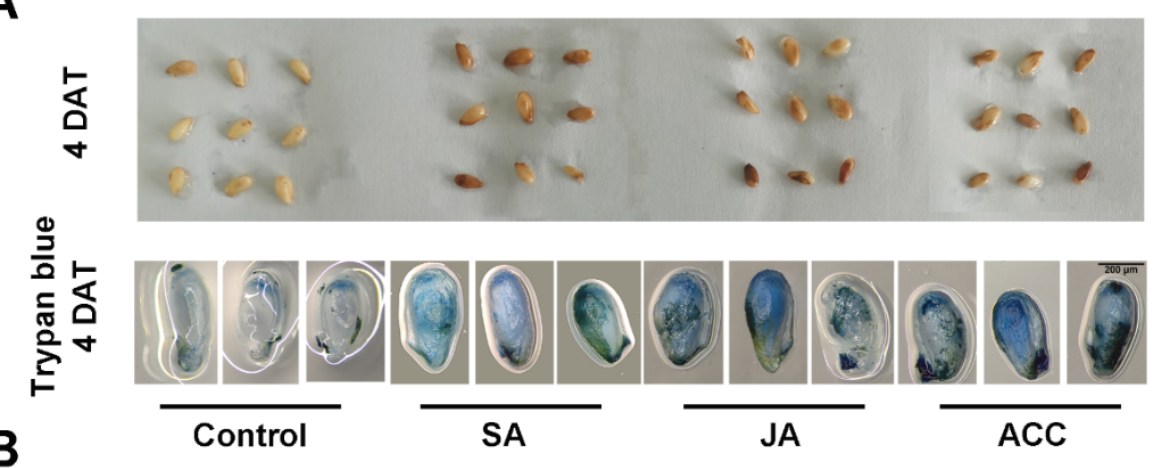

B
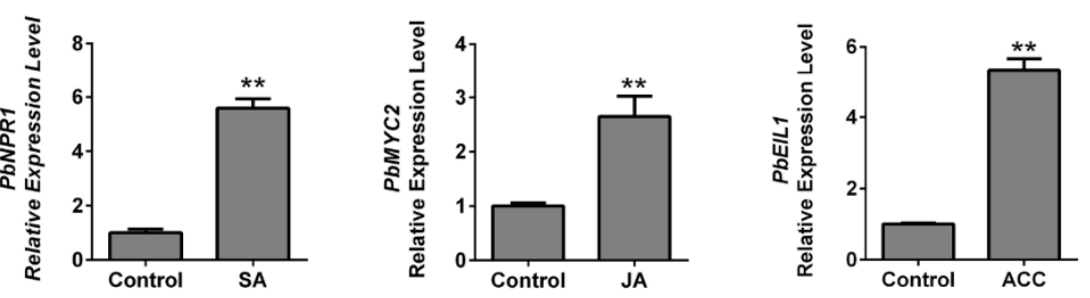

C
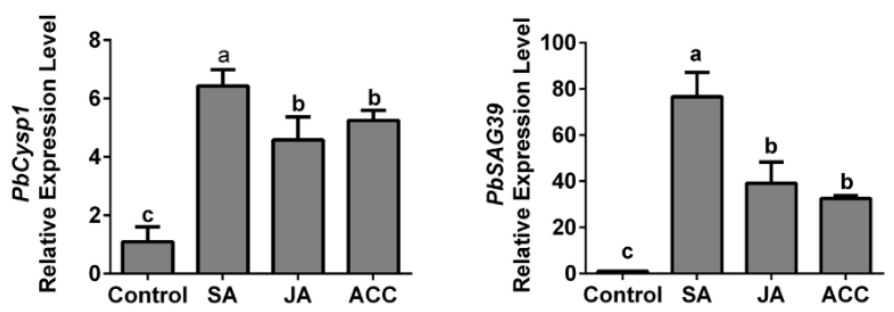

Figure 5. In vitro assay, application of ACC, SA and JA promotes normal seed death. (A) Observation of the seed development after treating with ACC, SA and JA respectively, and trypan blue staining assay demonstrated the cell death of different treated ovules. (B) Expression level analysis of the SA, JA and ethylene signaling response-related genes. (C) The expression of senescence-related genes $P b C y s p 1$ and $P b S A G 39$ verified the seed death. The data are mean $\pm S D$ of three biological replicates. Error bars represent the standard deviation. Asterisks indicate significant difference at $* * 00.01$ (Student's $t$-test). Different lowercase letters indicate significant difference at $p<0.05$ (Duncan's multiple range test).

\subsection{PbMYC2 Directly Triggers the Expression of PbSAG39}

In this study, JA and ethylene were found to be associated with the GA-triggered PCD process in the seedless pear ' 1913 '. Since the regulatory mechanism in which ethylene controls pear ovule PCD was elucidated in our previous study [4], it is clear that the JArelated mechanism controlling leaf senescence acts through MYC factors [8]. Consequently, we further investigated how JA participates in the PCD process of seedless pear ovules. MYC2 acts as a positive regulator of JA signaling to control downstream development. An analysis of the promoter sequences of ovule senescence-related genes $P b C y s p 1$ and PbSAG39 revealed typical G-box sequences in the promotors, which are MYC-binding sites. Expression profile analyses suggested that there were positive correlations among PbMYC2, PbCysp1 and PbSAG39 (Figure 5B,C). Using Y1H and dual-luciferase assays, 
we demonstrated that MYC2 directly bound the promoter of PbSAG39 and enhanced its expression, but this was not true for PbCysp1 (Figure 6). This suggests that JA participated in '1913' ovule death through MYC2's direct triggering of PbSGA39 expression, which leads to ovule death.
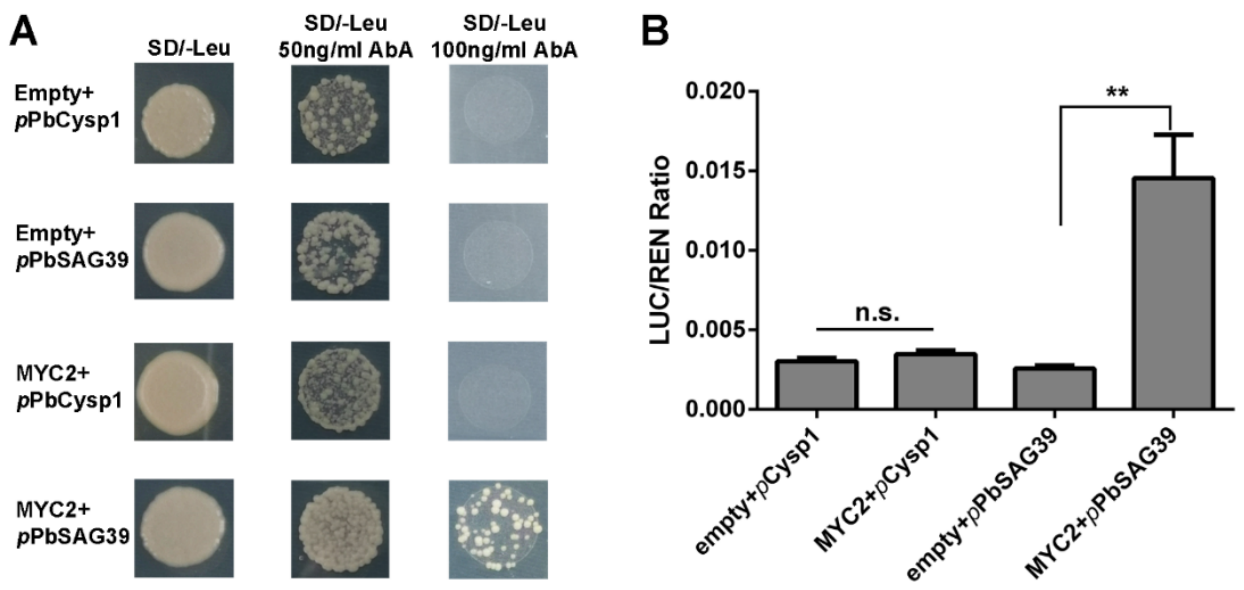

Figure 6. PbMYC2 promotion of PbSAG39 expression by direct binding to the PbSAG39 promoter. (A) Yeast one-hybrid assay verified the interaction of PbMYC2 with PbCysp1 and PbSAG39. (B) Validation of the activation effect of PbMYC2 on the PbSAG39 promoter based on a dual-luciferase assay. Relative promoter activity is represented by the ratio of the activity of the structural gene luciferase (LUC) to that of 35S Renilla (REN). An empty vector was used as a reference. Asterisks indicate significantly elevated LUC activity compared with that in the negative control ${ }^{* *} p<0.01$, Student's $t$-test). n.s., no significance.

\section{Discussion}

At the early stages of ovule development, the biosynthesis of various hormones, such as auxin and GA, is activated, and hormones play prominent roles in synchronizing fertilization and ovule growth [21]. In this study, we determined the roles of GA, JA, SA and ethylene in the control of ovule development and their relationships during ovule ontogenesis in seedless and seeded pear.

\subsection{Ovule Death Was Advanced in '1913' Pear by a $G A_{4+7}$ Treatment through Increased JA and} Ethylene Levels

Hormones play important roles in plants in the early developmental stages [25]. In plants, GAs are plant growth regulators controlling various aspects of plant growth and development [26]. A previous study reported that fertilization breaks the restrictions of ovule and fruit development in Arabidopsis, and during this process, it triggers the biosynthesis of GA in the ovules, which regulates ovule development [21]. This suggests that GAs play essential roles in ovule development, and evidence indicates that GAs potentially contribute to seed development. In this study, we found that ovule death was advanced in the seedless pear '1913' variety, but death was prevented in seeded pears after $\mathrm{GA}_{4+7}$ treatments, compared with solvent-treated ovules (Figure 1). We previously analyzed the death of '1913' ovules after pollination and determined that at 7 days after pollination, ovule death occurred, and cytological observations confirmed that the ovules were normal before pollination [4]. This result is similar to that of the $\mathrm{GA}_{4+7}$ treatment, which suggests that the ovule death of '1913' is triggered by external signals. Taken together, GA appears to activate different metabolic processes or developmental signaling in seedless and seeded pears, and it triggers the potential PCD process in '1913' ovules.

Next, we analyzed the contents of endogenous hormones in different samples and found that ACC, JA and SA may participate in the PCD process of '1913' ovules (Figure 2). By analyzing these relationships, we determined that JA and ethylene respond to $\mathrm{GA}_{4+7}$ treatments, but SA does not (Figure 2), and the expression pattern of their pathway-related 
genes also indicated their relationship with $\mathrm{GA}_{4+7}$ treatment (Figure 3). However, we found that $\mathrm{GA}_{4+7}$ treatments significantly increased the JA and ACC levels in the two seeded pear ovules, compared with the control (Figure 2), but the treatments did not cause ovule death as in the '1913' seedless pear. This may result from the positive regulatory roles of GAs on ovule development. Although the JA and ethylene levels increased in seeded pear ovules, their levels were lower than in '1913' ovules. Therefore, they were unable to cause ovule death. Moreover, in the relationship between GAs and JA, ethylene has also been reported. In Arabidopsis, GA promotes the expression of the JA biosynthetic gene DAD1 and JA biosynthesis, and through DELLAs, it regulates the expression levels of $M Y B 21 / 24 / 57$, which in turn promote stamen development [27]. In addition, GA activates the degradation of DELLAs, which allows JAZ1 to bind MYC2 and suppress the MYC2-dependent JAsignaling output [28]. Furthermore, FsACO1 expression is drastically increased in seeds after a $\mathrm{GA}_{3}$ treatment, but it is decreased by paclobutrazol, a GA biosynthetic inhibitor [29]. However, there are no clear reports regarding the GA-associated mechanism that regulates ethylene biosynthesis.

\subsection{JA, SA and Ethylene Involvement in Pear Ovule PCD}

Different types of PCD play crucial roles in plant reproductive and vegetative development, as well as in responses to environmental stresses. In this study, we found that a GA $_{4+7}$ treatment triggered potential PCD in '1913' ovules (Figure 1). Further analyses determined that JA, SA and ethylene were present at higher levels in '1913' than in seeded pears (Figure 2), and that applying JA, SA or ACC to normal 'Bartlett' ovules resulted in the promotion of ovule PCD (Figure 5).

The lipid-derived stress hormone JA regulates plant adaptations to biotic stresses [30], and it also regulates various aspects of development, including stamen and ovule development, root growth, flowering and leaf senescence [31-34]. In Arabidopsis, myc3 and myc4 plants have larger ovules and longer outer integuments than wild-type plants at 0 days after pollination, and $M Y C 3 / 4$ restrict cell proliferation and cell elongation in the ovules after fertilization [34]. Thus, JA signaling may regulate ovule development. In addition, JA regulates leaf senescence in a variety of plant species [35-38]. In Arabidopsis, MYC2 directly triggers the expression of $S A G 29$ to regulate leaf senescence [8]. Moreover, exogenous applications of methyl-JA promote leaf senescence in attached and detached leaves [35]. Thus, JA appears to have a senescence-promoting role. Although there are no clear reports on the functions of JA in ovule senescence, ovules derived from specialized meristematic regions within the carpels, referred to as the placentas, are proposed to have evolved from ancestral foliar organs, either leaf-like sporophylls that folded to enclose the ovules or bract-like structures that subtend shoot-like ovules [17]. This evidence suggests that JA may regulate ovule senescence and have a similar regulatory mechanism to leave senescence. In this study, we found that an exogenous application of JA promoted ovule senescence (Figure 5) and that MYC2 triggered the expression of PbSAG39, leading to ovule senescence (Figure 6).

In plants, ethylene, JA, auxin and strigolactones have been implicated in developmentcontrolled PCD signaling $[8,9,39]$. Among them, ethylene is the best-characterized dPCD phytohormone [7]. In Arabidopsis, fertilization triggers the ethylene signaling that contributes to the elimination of the persistent synergids via cell fusion and nuclear degradation, which terminates pollen tube attraction [11,40]. In Zinnia elegans, the chemical inhibition of ethylene signaling delays xylem differentiation but directly blocks PCD [41]. Ethylene is also involved in pistil fate by modulating the onset of ovule senescence [14]. In our previous study, we reported that ethylene over-accumulates in '1913' ovules after pollination and that the ethylene signaling acceptor PbEIL1 directly activates the expression of $P b C y s p 1$ [4]. Thus, we have determined that ethylene participates in the PCD process of '1913' ovules and that ethylene controls downstream PCD execution. Thus, we have determined that ethylene participates in the $\mathrm{GA}_{4+7}$-induced PCD process of '1913' ovules, 
but we did not conduct research on how the regulatory mechanism of ethylene affects '1913' ovule abortion in this study.

As the only hormone strictly required for the establishment of pathogen-triggered PCD (pPCD), SA promotes pPCD, leading to susceptibility to necrotrophs and biotrophs [42,43]. In addition, SA regulates flower formation, seed germination, embryo development and senescence. In this study, we found that SA was present at a higher level in seedless pear ovules than in seeded pear ovules, but there was no significant difference in its level between $\mathrm{GA}_{4+7}$-treated and control groups (Figures 2 and 3). Royo et al. reported that SA has potential roles in promoting grape seed abortion [44]. Additionally, the SA content is higher in seedless 'Thompson' grapes than in seeded grapes, and HDZ28 positively regulates SA biosynthesis, leading to seed abortion [25]. Thus, SA appears to play roles in the PCD progress and is associated with seed abortion in seeded plants. In our results, although SA did not respond to the $\mathrm{GA}_{4+7}$ treatment of ovules (Figures 2 and 3), the exogenous spraying of $\mathrm{SA}$ induced the expression of the senescence-related genes $\mathrm{PbCysp} 1$ and PbSAG39 and increased 'Bartlett' seed death (Figure 5). This suggests that SA is involved in '1913' seed abortion. Further studies will be conducted to determine the regulatory mechanism.

\section{Materials and Methods}

\subsection{Plant Materials and Treatments}

The samples used in study were collected from a pear orchard in Meixian, Shaanxi, China, in $2021\left(34.29^{\circ} \mathrm{N}, 107.76^{\circ} \mathrm{E}, 514 \mathrm{~m}\right.$ above sea level) [45]. The average annual precipitation at this location was $574.6 \mathrm{~mm}$, and the average annual temperature was $12.7^{\circ} \mathrm{C}$. The average temperature was $12^{\circ} \mathrm{C}$ during anthesis.

In this study, seedless cultivar '1913' pear (Pyrus hybrid), and seeded cultivars 'Dangshansu' pear (Pyrus bretschneideri Rehd.) and 'Bartlett' (Pyrus communis L.), were selected as materials. Flowering of the three varieties occurs in late March. The pear cultivar '1913' is a hybrid selected from a cross of 'Bartlett' with a male parent of 'Zaosu', a hybrid of 'Shenbuzhi' (Pyrus communis L.) and 'Pingguoli' (Pyrus bretschneideri Rehd.). For '1913', it has been reported in our previous study that the block of fertilization cause ovule abortion [4]. The seedless characteristic of '1913' has proven stable over many years of observation. Two days before flowering, all selected materials were bagged to prohibit pollination. Healthy and uniform plants were subjected to two treatments: (i) application of water, containing $0.1 \%$ ethanol $(v / v)$ on unpollinated flowers, serving as the control, and (ii) spraying a solution of $50 \mathrm{mg} \mathrm{L}^{-1}$ of $\mathrm{GA}_{4+7}$ on unpollinated flowers, where the concentration was determined by Wang et al. [45]. Each treatment consisted of 200 inflorescences. The same treatment was performed in the three pear varieties. Samples were collected at 0,8 and 16 days after treatment (DAT). Several samples were used for observation of ovule development, which were examined under a microscope (MZ10F, Leica, Wetzlar, Germany). The other samples were collected and the ovules were separated from it quickly, then the ovules samples were divided into two groups, including the complete ovules group and the separated ovules group for different experiments. The remaining samples were immediately frozen in liquid nitrogen and stored at $-80^{\circ} \mathrm{C}$ for total RNA extraction.

\subsection{Fluorescein Diacetate (FDA) and Trypan Blue Staining}

For the FDA staining assay, it was performed as described previously, with minor modifications [46]. Briefly, fresh sample was cut into $1 \mathrm{~mm}$ in the longitudinal direction, and washed with phosphate buffer saline (PBS) 3 times, then the sample was immersed in FDA working solution: a stock solution of FDA (Coolaber, Beijing, China) was prepared by dissolving $5 \mathrm{mg} \mathrm{mL}^{-1}$ in acetone, then the samples were stained for $10 \mathrm{~min}$ with $12.5 \mu \mathrm{g}$ $\mathrm{mL}^{-1}$ FDA, which was diluted with deionized water. After the extra dye was removed by washing with deionized water for $5 \mathrm{~min}$ and repeating 3 times, the ovule sample was observed under a laser scanning confocal microscope (TCS-SP8 SR, Leica, Wetzlar, Germany). Excitation and emission wavelengths were 485 and $530 \mathrm{~nm}$, respectively. 
For trypan blue staining, the method was performed as described previously, with minor modifications [47]. For staining, the samples were immersed in boiled lactophenol (glycerol:lactic acid:liquid phenol:distilled water, 1:1:1:1) with $0.25 \mathrm{mg} \mathrm{mL}^{-1}$ of trypan blue for $10 \mathrm{~min}$. Then, the samples were de-stained with $2.5 \mathrm{~g} \mathrm{~mL}^{-1}$ of chloral hydrate aqueous solution for at least $36 \mathrm{~h}$. Next, the sample was observed under a microscope (SZX16, OLYMPUS, Tokyo, Japan).

\section{3. qRT-PCR Validation of Gene Expression Levels}

Total RNA was extracted from the ovule sample by using an RNAprep Pure Plant Kit (Tiangen, Beijing, China). Then, $1 \mu \mathrm{g}$ of total RNA was reverse-transcribed into cDNA following the manufacturer's instructions of the PrimeScript RT Reagent Kit with a gDNA Eraser (Takara, Dalian, China). Next, the qRT-PCR assay was used to analyze the expression of all detected genes. Sequence similarities were examined based on Pyrus bretschneideri genome data, and gene-specific primers (Supplementary Table S1) were designed using Primer 5 software. Then, RT-PCR and melting curve analysis were used to determine the primer specificity. The qRT-PCR assay was performed on an ABI StepOnePlus Real-Time PCR System (Thermo Fisher Scientific, Waltham, MA, USA) using TB Green Premix Ex Taq II (Tli RNaseH Plus, Takara, Dalian, China). Transcripts of PbActin7 (LOC103926846) served to standardize the cDNA from our test genes. For each sample, three biological replicates were used in the assay. For data analysis, the relative expression level of each gene was calculated using the cycle threshold (Ct) $2^{-\Delta \Delta \mathrm{Ct}}$ method [48].

\subsection{Phytohormone Detection}

Concentrations of endogenous hormones, including $\mathrm{GA}_{4}$, aminocyclopropane-1carboxylic acid (ACC, the precursor of ethylene), JA, SA, IAA, ABA and cytokinins (transzeatin and trans-zeatin riboside), in ovules of '1913', 'Bartlett' and 'Dangshansu', were extracted as described previously [4]. The detailed method is referred to in our previous study [4].

\subsection{Application of JA, SA and ACC on Ovules In Vitro}

Fruit of 'Bartlett' were collected at 30 days after pollination, and then ovules were separated and precultured in a Petri dish with PBS-moistened filter paper in the dark. After culturing for $12 \mathrm{~h}$, the ovules with black or brown surfaces were removed, and the rest of the ovules were used for treatments. Water treatment was used as a control, and ovules were also treated with $10 \mu \mathrm{M}$ ACC [49], $50 \mu \mathrm{M} \mathrm{JA}$ and $250 \mu \mathrm{M} \mathrm{SA}$ [50], respectively. The development of different treated groups was observed, and the ovules were stained at significant difference stages by trypan blue.

\subsection{Yeast One-Hybrid Assay (Y1H)}

The full-length coding sequence of $P b M Y C 2$ was amplified and inserted into the MCS of pGADT7 AD, and the specific truncated promoter sequence fragment of $P b C y s p 1$ and PbSAG39 was inserted into pAbAi bait vectors. Then, the method of the interaction assay was performed according to Wang et al. [4]. The related primers are listed in Supplementary Table S1.

\subsection{Dual-Luciferase Assay}

The full-length coding sequence of $P b M Y C 2$ was amplified and inserted into the MCS region of a pGreenII 0029 62-SK binary vector (effector vector), and the promoter sequences of $P b C y s p 1$ and PbSAG39 was inserted into the dual-LUC plasmid pGreenII 0800-LUC (reporter vector). The methods of transformation and result analyses were carried out according to Hellens et al. [51] and Wang et al. [4]. 


\subsection{Statistical Analysis}

Data were subjected to analysis of variance and tested for significant ${ }^{*} p<0.05$, $\left.{ }^{* *} p<0.01\right)$ treatment differences using Duncan's test and Student's $t$-test. The results are presented as mean \pm standard deviation (SD) of three replicate samples.

\section{Conclusions}

In this study, we demonstrated that $\mathrm{GA}_{4+7}$ induces ovule abortion in advance by increasing the endogenous level of JA and ethylene, and SA is also associated with ovule death, though it does not respond to $\mathrm{GA}_{4+7}$ treatment. We previously demonstrated that ethylene regulates pear ovule death through PbEIL1, and directly triggers the expression of the ovule senescence-related gene $P b C y s p 1$, and in this study, we further studied the regulation of JA on ovule death, whereby JA participates in pear death through PbMYC2, binds the promoter of PbSAG39 and increases its expression, leading to ovule death (Figure 7).
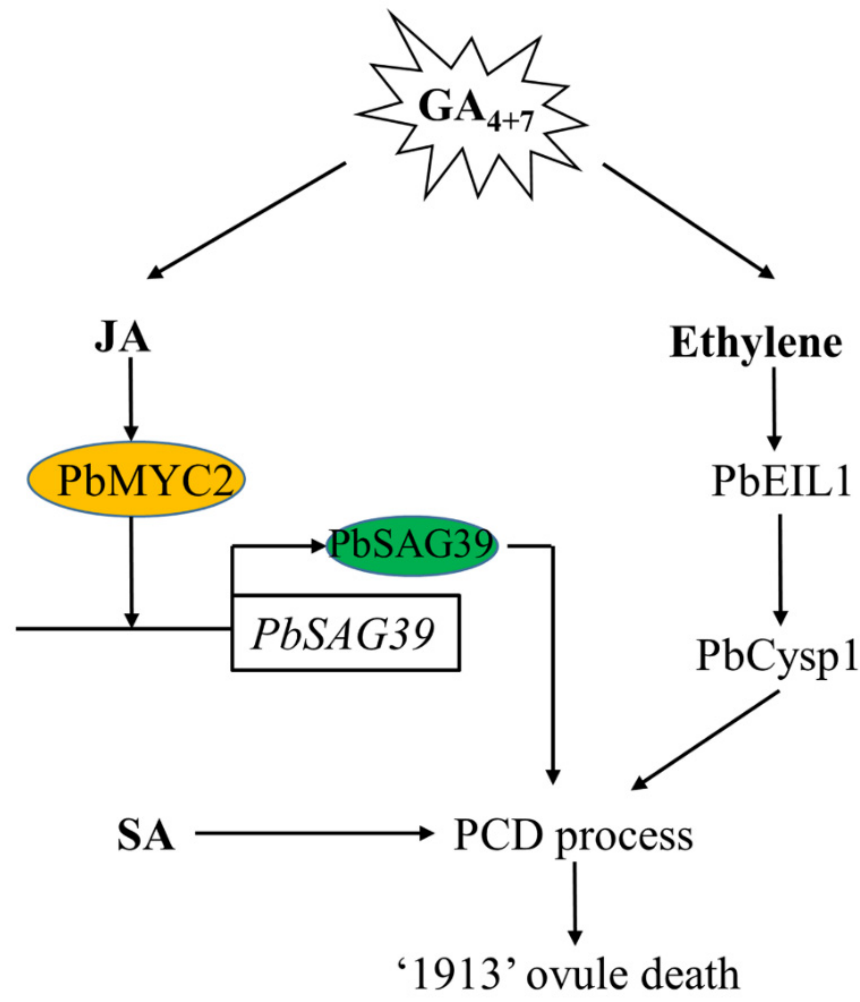

Figure 7. Model depicting the mechanism of $\mathrm{GA}_{4+7}$-induced seedless pear ovule abortion in advance. $\mathrm{GA}_{4+7}$ treatment increases the production of JA and ethylene, and JA and ethylene signaling receptors PbMYC2 and PbEIL1 directly enhance their target genes PbSGA39 and PbCysp1 to regulate pear ovule abortion, respectively. SA also participates in the pear ovule PCD process. $\mathrm{GA}_{4+7}$, Gibberellin A4 and A7; JA, Jasmonic Acid; SA, Salicylic acid; PCD, Programmed Cell Death.

Supplementary Materials: The following are available online at https:/ /www.mdpi.com/article/10 .3390/ijms22189844/s1.

Author Contributions: Conceptualization, H.W.; Investigation, H.W., S.Z., Y.Q., R.G. and Y.X.; Formal analysis, H.W., Z.W., R.Z., C.Y. and L.X.; Resources, L.X.; Writing-original draft preparation, H.W.; Writing-review and editing, H.W. and L.X.; Funding acquisition, L.X. All authors have read and agreed to the published version of the manuscript.

Funding: This research was funded by China Agriculture Research System of MOF and MARA (CARS-28-46).

Institutional Review Board Statement: Not applicable. 
Informed Consent Statement: Not applicable.

Data Availability Statement: All data generated and analyzed during this study are included in this published article. The ethics approval and consent to participate are not applicable.

Conflicts of Interest: All the authors declare that they have no competing interests.

\section{References}

1. Lo'ay, A.A.; El-Boray, M.S. Improving fruit cluster quality attributes of 'Flame Seedless' grapes using preharvest application of ascorbic and salicylic acid. Sci. Hortic. 2018, 233, 339-348. [CrossRef]

2. Ma, Y.; Li, Q.; Hu, G.; Qin, Y. Comparative transcriptional survey between self-incompatibility and self-compatibility in Citrus reticulata Blanco. Gene 2017, 609, 52-61. [CrossRef] [PubMed]

3. Wijesinghe, S.A.E.C.; Evans, L.J.; Kirkland, L.; Rader, R. A global review of watermelon pollination biology and ecology: The increasing importance of seedless cultivars. Sci. Hortic. 2020, 271, 109493. [CrossRef]

4. Wang, H.; Zhang, H.; Liang, F.; Cong, L.; Song, L.; Li, X.; Zhai, R.; Yang, C.; Wang, Z.; Ma, F.; et al. PbEIL1 acts upstream of PbCysp1 to regulate ovule senescence in seedless pear. Hortic. Res. 2021, 8, 59. [CrossRef] [PubMed]

5. Daneva, A.; Gao, Z.; Van Durme, M.; Nowack, M.K. Functions and regulation of programmed cell death in plant development. Annu. Rev. Cell Dev. Biol. 2016, 32, 441-468. [CrossRef]

6. Trobacher, C.P. Ethylene and programmed cell death in plants. Botany 2009, 87, 757-769. [CrossRef]

7. Huysmans, M.; Lema, A.S.; Coll, N.S.; Nowack, M.K. Dying two deaths-programmed cell death regulation in development and disease. Curr. Opin. Plant Biol. 2017, 35, 37-44. [CrossRef] [PubMed]

8. Qi, T.; Wang, J.; Huang, H.; Liu, B.; Gao, H.; Liu, Y.; Song, S.; Xie, D. Regulation of Jasmonate-induced leaf senescence by antagonism between bHLH subgroup IIIe and IIId factors in Arabidopsis. Plant Cell 2015, 27, 1634-1649. [CrossRef]

9. Ueda, H.; Kusaba, M. Strigolactone regulates leaf senescence in concert with ethylene in Arabidopsis. Plant Physiol. 2015, 169, 138-147. [CrossRef]

10. Sarwat, M.; Naqvi, A.R.; Ahmad, P.; Ashraf, M.; Akram, N.A. Phytohormones and microRNAs as sensors and regulators of leaf senescence: Assigning macro roles to small molecules. Biotechnol. Adv. 2013, 31, 1153-1171. [CrossRef]

11. Volz, R.; Heydlauff, J.; Ripper, D.; von Lyncker, L.; Gross-Hardt, R. Ethylene signaling is required for synergid degeneration and the establishment of a pollen tube block. Dev. Cell. 2013, 25, 310-316. [CrossRef] [PubMed]

12. Lombardi, L.; Mariotti, L.; Picciarelli, P.; Ceccarelli, N.; Lorenzi, R. Ethylene produced by the endosperm is involved in the regulation of nucellus programmed cell death in Sechium edule Sw. Plant Sci. 2012, 187, 31-38. [CrossRef] [PubMed]

13. Young, T.E.; Callie, D.R.; DeMason, D.A. Ethylene-mediated programmed cell death during maize endosperm development of wild-type and shrunken2 genotypes. Plant Physiol. 1997, 115, 737-751. [CrossRef] [PubMed]

14. Carbonell-Bejerano, P.; Urbez, C.; Granell, A.; Carbonell, J.; Perez-Amador, M.A. Ethylene is involved in pistil fate by modulating the onset of ovule senescence and the GA-mediated fruit set in Arabidopsis. BMC Plant Biol. 2011, 11, 84. [CrossRef] [PubMed]

15. Major, I.T.; Yoshida, Y.; Campos, M.L.; Kapali, G.; Xin, X.F.; Sugimoto, K.; de Oliveira Ferreira, D.; He, S.Y.; Howe, G.A. Regulation of growth-defense balance by the Jasmonate Zim-Domain (JAZ)-MYC transcriptional module. New Phytol. 2017, 215, 1533-1547. [CrossRef]

16. Yang, J.; Duan, G.; Li, C.; Liu, L.; Han, G.; Zhang, Y.; Wang, C. The crosstalks between Jasmonic acid and other plant hormone signaling highlight the involvement of Jasmonic acid as a core component in plant response to biotic and abiotic stresses. Front. Plant Sci. 2019, 10, 1349. [CrossRef]

17. Skinner, D.J.; Hill, T.A.; Gasser, C.S. Regulation of ovule development. Plant Cell 2004, 16, S32-S45. [CrossRef]

18. Coll, N.S.; Epple, P.; Dangl, J.L. Programmed cell death in the plant immune system. Cell Death Differ. 2011, 18, 1247-1256. [CrossRef] [PubMed]

19. Lim, P.O.; Kim, H.J.; Nam, H.G. Leaf senescence. Annu. Rev. Plant Biol. 2007, 58, 115-136. [CrossRef]

20. Buchanan-Wollaston, V.; Page, T.; Harrison, E.; Breeze, E.; Lim, P.O.; Nam, H.G.; Lin, J.F.; Wu, S.H.; Swidzinski, J.; Ishizaki, K.; et al. Comparative transcriptome analysis reveals significant differences in gene expression and signalling pathways between developmental and dark/starvation-induced senescence in Arabidopsis. Plant J. 2005, 42, 567-585. [CrossRef] [PubMed]

21. Dorcey, E.; Urbez, C.; Blazquez, M.A.; Carbonell, J.; Perez-Amador, M.A. Fertilization-dependent auxin response in ovules triggers fruit development through the modulation of gibberellin metabolism in Arabidopsis. Plant J. 2009, 58, 318-332. [CrossRef]

22. Singh, D.P.; Jermakow, A.M.; Swain, S.M. Gibberellins are required for seed development and pollen tube growth in Arabidopsis. Plant Cell 2002, 14, 3133-3147. [CrossRef]

23. Swain, S.M.; Reid, J.B.; Kamiya, Y. Gibberellins are required for embryo growth and seed development in pea. Plant J. 1997, 12, 1329-1338. [CrossRef]

24. Wan, L.L.; XIA, Q.; Qiu, X.; Selvaraj, G. Early stages of seed development in Brassica napus: A seed coat-specific cysteine proteinase associated with programmed cell death of the inner integument. Plant J. 2002, 30, 1-10. [CrossRef] [PubMed]

25. Li, Z.; Jiao, Y.; Zhang, C.; Dou, M.; Weng, K.; Wang, Y.; Xu, Y. VvHDZ28 positively regulate salicylic acid biosynthesis during seed abortion in Thompson Seedless. Plant Biotechnol. J. 2021, 13596. [CrossRef]

26. Swain, S.M.; Singh, D.P. Tall tales from sly dwarves: Novel functions of gibberellins in plant development. Trends Plant Sci. 2005, 10, 123-129. [CrossRef] 
27. Cheng, H.; Song, S.; Xiao, L.; Soo, H.M.; Cheng, Z.; Xie, D.; Peng, J. Gibberellin acts through jasmonate to control the expression of MYB21, MYB24, and MYB57 to promote stamen filament growth in Arabidopsis. PLoS Genet. 2009, 5, e1000440. [CrossRef]

28. Hou, X.; Lee, L.Y.; Xia, K.; Yan, Y.; Yu, H. DELLAs modulate Jasmonate signaling via competitive binding to JAZs. Dev. Cell. 2010, 19, 884-894. [CrossRef]

29. Calvo, A.P.; Nicolás, C.; Nicolás, G.; Rodríguez, D. Evidence of a cross-talk regulation of a GA 20-oxidase (FsGA20ox1) by gibberellins and ethylene during the breaking of dormancy in Fagus sylvatica seeds. Physiol. Plant. 2004, 120, 623-630. [CrossRef]

30. Huang, H.; Liu, B.; Liu, L.; Song, S. Jasmonate action in plant growth and development. J. Exp. Bot. 2017, 68, 1349-1359. [CrossRef] [PubMed]

31. Goossens, J.; Fernandez-Calvo, P.; Schweizer, F.; Goossens, A. Jasmonates: Signal transduction components and their roles in environmental stress responses. Plant Mol. Biol. 2016, 91, 673-689. [CrossRef] [PubMed]

32. Howe, G.A.; Jander, G. Plant immunity to insect herbivores. Annu. Rev. Plant Biol. 2008, 59, 41-66. [CrossRef] [PubMed]

33. Wasternack, C.; Hause, B. Jasmonates: Biosynthesis, perception, signal transduction and action in plant stress response, growth and development. An update to the 2007 review in Annals of Botany. Ann. Bot. 2013, 111, 1021-1058. [CrossRef]

34. Liu, Z.; Li, N.; Zhang, Y.; Li, Y. Transcriptional repression of GIF1 by the KIX-PPD-MYC repressor complex controls seed size in Arabidopsis. Nat. Commun. 2020, 11, 1846. [CrossRef] [PubMed]

35. He, Y.; Fukushige, H.; Hildebrand, D.F.; Gan, S. Evidence supporting a role of Jasmonic acid in Arabidopsis leaf senescence. Plant Physiol. 2002, 128, 876-884. [CrossRef]

36. Yan, J.; Zhang, C.; Gu, M.; Bai, Z.; Zhang, W.; Qi, T.; Cheng, Z.; Peng, W.; Luo, H.; Nan, F.; et al. The Arabidopsis Coronatine insensitive1 protein is a Jasmonate receptor. Plant Cell 2009, 21, 2220-2236. [CrossRef]

37. Hu, Y.; Jiang, Y.; Han, X.; Wang, H.; Pan, J.; Yu, D. Jasmonate regulates leaf senescence and tolerance to cold stress: Crosstalk with other phytohormones. J. Exp. Bot. 2017, 68, 1361-1369. [CrossRef]

38. Lingam, S.; Mohrbacher, J.; Brumbarova, T.; Potuschak, T.; Fink-Straube, C.; Blondet, E.; Genschik, P.; Bauer, P. Interaction between the bHLH transcription factor FIT and Ethylene Insensitive3/Ethylene Insensitive3-Like1 reveals molecular linkage between the regulation of iron acquisition and ethylene signaling in Arabidopsis. Plant Cell 2011, 23, 1815-1829. [CrossRef]

39. Yin, L.; Xue, H. The MADS29 transcription factor regulates the degradation of the nucellus and the nucellar projection during rice seed development. Plant Cell 2012, 24, 1049-1065. [CrossRef]

40. Maruyama, D.; Volz, R.; Takeuchi, H.; Mori, T.; Igawa, T.; Kurihara, D.; Kawashima, T.; Ueda, M.; Ito, M.; Umeda, M.; et al. Rapid elimination of the persistent synergid through a cell fusion mechanism. Cell 2015, 161, 907-918. [CrossRef] [PubMed]

41. Pesquet, E.; Zhang, B.; Gorzsas, A.; Puhakainen, T.; Serk, H.; Escamez, S.; Barbier, O.; Gerber, L.; Courtois-Moreau, C.; Alatalo, E.; et al. Non-cell-autonomous postmortem lignification of tracheary elements in Zinnia elegans. Plant Cell 2013, 25, 1314-1328. [CrossRef]

42. Birkenbihl, R.P.; Somssich, I.E. Transcriptional plant responses critical for resistance towards necrotrophic pathogens. Front. Plant Sci. 2011, 2, 76. [CrossRef]

43. Pieterse, C.M.; Leon-Reyes, A.; Van der Ent, S.; Van Wees, S.C. Networking by small-molecule hormones in plant immunity. Nat. Chem. Biol. 2009, 5, 308-316. [CrossRef]

44. Royo, C.; Torres-Perez, R.; Mauri, N.; Diestro, N.; Cabezas, J.A.; Marchal, C.; Lacombe, T.; Ibanez, J.; Tornel, M.; Carreno, J.; et al. The major origin of seedless grapes is associated with a missense mutation in the MADS-Box Gene VviAGL11. Plant Physiol. 2018, 177, 1234-1253. [CrossRef]

45. Wang, H.; Wu, T.; Liu, J.; Cong, L.; Zhu, Y.; Zhai, R.; Yang, C.; Wang, Z.; Ma, F.; Xu, L. PbGA20ox2 regulates fruit set and induces parthenocarpy by enhancing $\mathrm{GA}_{4}$ content. Front. Plant Sci. 2020, 11, 113. [CrossRef]

46. Ishikawa, S.; Wagatsuma, T. Plasma membrane permeability of root-tip cells following temporary exposure to al ions is a rapid measure of Al tolerance among plant species. Plant Cell Physiol. 1998, 39, 516-525. [CrossRef]

47. Koch, E.; Slusarenko, A. Arabidopsis is Susceptible to lnfection by a Downy Mildew Fungus. Plant Cell 1990, 2, 437-445. [PubMed]

48. Livak, K.J.; Schmittgen, T.D. Analysis of relative gene expression data using real-time quantitative PCR and the 2(- $\Delta \Delta \mathrm{C}(\mathrm{T}))$ method. Methods 2001, 25, 402-408. [CrossRef] [PubMed]

49. Vanderstraeten, L.; Depaepe, T.; Bertrand, S.; Van Der Straeten, D. The ethylene precursor ACC affects early vegetative development independently of Ethylene signaling. Front. Plant Sci. 2019, 10, 1591. [CrossRef] [PubMed]

50. Seo, J.; Yi, G.; Lee, J.G.; Choi, J.H.; Lee, E.J. Seed browning in pepper (Capsicum annuum L.) fruit during cold storage is inhibited by methyl Jasmonate or induced by methyl salicylate. Postharvest Biol. Technol. 2020, 166, 111210. [CrossRef]

51. Hellens, R.; Allan, A.; Friel, E.; Bolitho, K.; Grafton, K.; Templeton, M.; Karunairetnam, S.; Gleave, A.; Laing, W. Transient expression vectors for functional genomics, quantification of promoter activity and RNA silencing in plants. Plant Methods 2005, 1, 13. [CrossRef] [PubMed] 